\title{
Sciences et techniques dans l'espace scolaire
}

Quelques réflexions sociologiques

\section{Polymnia Zagefka}

\section{CpenEdition}

\section{Journals}

Édition électronique

URL : http://journals.openedition.org/ries/3355

DOI : $10.4000 /$ ries.3355

ISSN : 2261-4265

Éditeur

Centre international d'études pédagogiques

Édition imprimée

Date de publication : 1 juin 1997

Pagination : 85-90

ISSN : 1254-4590

Référence électronique

Polymnia Zagefka, «Sciences et techniques dans l'espace scolaire », Revue internationale d'éducation de Sèvres [En ligne], 14 | 1997, mis en ligne le 30 juillet 2013, consulté le 01 mai 2019. URL : http:// journals.openedition.org/ries/3355; DOI : 10.4000/ries.3355

Ce document a été généré automatiquement le 1 mai 2019.

(c) Tous droits réservés 


\title{
Sciences et techniques dans l'espace scolaire
}

\author{
Quelques réflexions sociologiques
}

Polymnia Zagefka

1 Les exhortations coordonnées en faveur d'une «culture scientifique et technique » du début des années quatre-vingt ont abouti à la création de nouvelles sphères d'activités liées à la "vulgarisation » et à la diffusion des sciences et des techniques, en association étroite avec des chercheurs d'origines disciplinaires diverses. Principales institutions de ce renouveau, les musées des sciences ont accordé une attention soutenue à une catégorie particulière de leur public potentiel : les enfants et les adolescents non seulement dans le cadre des visites familiales "privées" mais encore les "scolaires", pour reprendre le terme couramment utilisé, ce qui implique une coordination institutionnelle relativement complexe et une redéfinition des compétences professionnelles des enseignants ${ }^{1}$.

2 C'est la même période qui voit l'émergence et la confirmation de deux nouveaux champs de recherche; en premier lieu, la didactique des disciplines scolaires, fortement ancrée dans les savoirs disciplinaires spécifiques, qu'il s'agisse des mathématiques, de la physique ou de la biologie ${ }^{2}$. En second lieu, l'histoire des disciplines scolaires, grâce notamment aux travaux de l'équipe d'histoire de l'éducation de l'INRP. Quant à la sociologie des savoirs scolaires ou "sociologie du curriculum ", elle acquiert lentement au cours de la même période une nouvelle identité à travers la "rencontre", certes décalée, des travaux menés autour de Viviane Isambert-Jamati et de Lucie Tanguy avec ceux produits en milieu anglo-saxon dans le cadre de la "nouvelle sociologie de l'éducation ».

3 Dans ce contexte général, aussi bien institutionnel que théorique, qu'en est-il de l'éducation scientifique aujourd'hui? Peut-on comprendre l'enseignement des savoirs scientifiques et techniques sans interroger avant tout les spécificités de l'institution scolaire? 


\section{L'usage des termes}

4 Pour ce qui concerne les « connaissances » ou les « savoirs » nous disposons actuellement en France d'une série des termes introduits successivement: "connaissances », terme ayant une connotation noble, car il désigne l'acte de la pensée qui pose un objet en tant qu'objet, qui le pénètre et le définit en tant qu'objet de sa connaissance, la chose connue et le contenu de la connaissance. Ce terme est employé depuis une vingtaine d'années de moins en moins, au profit du terme «savoirs» (accompagné de ses extensions, telles "savoir-faire » et plus rarement "savoir-être ») que l'on spécifie fréquemment; ainsi, nous sommes amenés à parler de "savoirs scolaires", de "savoirs scientifiques" ou "universitaires" au même titre que de "savoirs professionnels" ou de "savoirs techniques $»^{3}$. Tout en n'ignorant pas que des déplacements linguistiques de cet ordre puissent être un effet de mode - le néologisme faisant souvent figure d'innovation conceptuelle -, toujours est-il que l'étendue de son utilisation démontre le changement social sous-jacent à la segmentation institutionnelle définissant le champ de connaissances et dont on fait souvent l'économie de questionner la signification. Plus récemment encore, comme viennent de nous le montrer les travaux de L. Tanguy et $\mathrm{F}$. Ropé 4 , le terme "compétence " supplante celui de "savoirs » qui, malgré son usage polysémique, met l'accent sur l'applicabilité individuelle des connaissances normalisées et transférables, en minimisant les contenus, fussent-ils circonscrits à des secteurs précis d'activité.

5 En milieu anglo-saxon, au contraire, il n'existe qu'un terme générique, celui de knowledge, qui englobe « connaissances » et « savoirs », voire, même dans certains cas, les « savoirs et compétences ouvriers » (skill), même si certaines distinctions ont été élaborées afin d'affiner telle ou telle acception du terme. Ainsi trouvons-nous l'opposition entre knowledge et knowledge about qui serait plus proche de "savoirs » et une assimilation forte entre knowledge et académic (disciplines d'enseignement général) s'opposant par là à vocational, tandis que les "compétences" sont définies en "termes de performances observables $»^{5}$. Pour ce qui concerne l'institution scolaire nous trouvons alors le terme school knowledge.

6 Quant aux «sciences et techniques » et a fortiori à la «technologie », les termes utilisés recouvrent des significations variées selon les perspectives adoptées par les différents auteurs. Il n'y a pas lieu ici d'évoquer les débats autour de la définition de ces termes qui renvoient globalement aux positions sociales différentielles de leurs émetteurs respectifs ${ }^{6}$ - Retenons toutefois que les disciplines définies institutionnellement dans l'espace scolaire, en dépit de leurs variations dans le temps et selon les pratiques professionnelles concrètes, sont la plupart du temps adoptées comme des catégories « naturelles ». Ainsi le découpage institutionnel actuellement en vigueur propose entre autres les «mathématiques", les «sciences de la nature », les «sciences physiques» mais aussi la "technologie", découpage largement adopté par les recherches ancrées dans les disciplines. Par ailleurs, un débat, qui se poursuit encore aujourd'hui, se pose comme une alternative entre « enseigner les sciences, en l'occurrence, la physique » ou « enseigner la technologie $\aleph^{7}$. Formulée ainsi, l'alternative adopte le découpage institutionnel des disciplines enseignées et fonde de nouveau une opposition entre les deux branches des savoirs. Or, précisément, la pertinence de cette opposition est fortement questionnée par les développements récents de la recherche scientifique comme c'est le cas, par exemple, 
de la supra-conductivité à haute température ou celui de l'usage du rayonnement synchrotron.

7 En dépit du découpage disciplinaire en vigueur, les termes combinés «sciences et techniques » ou « savoirs scientifiques et techniques » me semblent plus pertinents. Non seulement parce qu'une distinction nette est hasardeuse, pouvant à la limite donner lieu à d'innombrables exégèses de type spéculatif ou, plus concrètement, à des conflits des compétences professionnelles au sein du corps enseignant. Mais encore et surtout, pour souligner le haut degré de technicité inscrit dans le développement historique de la plupart des sciences, technicité dont il faudrait voir dans quelle mesure et sous quelle forme elle s'intègre dans la transmission et dans l'apprentissage de celles-ci.

\section{Procédés et démarches ostensibles}

Une des dimensions structurant la sphère des savoirs scolaires est le caractère ostensible des performances orales ou écrites aussi bien des enseignants que des élèves. La place accordée à la "démonstration » dans le cadre de l'enseignement des mathématiques en est un exemple marquant; et les didacticiens, comme Y. Chevallard, A. Mercier ou S. Coppé ont bien mis en évidence que les élèves construisent et entretiennent un rapport particulier avec cet objet. Tout en étant une dimension spécifique à la discipline des mathématiques, son organisation, comme celle particulière à d'autres disciplines scolaires, est soumise à certaines règles censées être construites et intériorisées au fil de plusieurs années d'apprentissage. Ces règles, rappelons-le, participent d'un ordre plus large, le plus souvent implicite, d'acquisition de compétences scolaires ${ }^{8}$. Les élèves, ou certains parmi eux, apprennent à reconnaître et à appliquer les règles d'une "bonne démonstration » qui doit en plus être selon la tradition française des mathématiques une "démonstration élégante». Les règles relatives à la "démonstration» renvoient en partie à la dimension "d'ostension» du travail de l'élève, appréhendée et critiquée à juste titre par A. Mercier. Le rapport de l'élève à la "démonstration" condense l'obligation scolaire de matérialiser et de «faire montrer " l'activité d'apprentissage. Malgré l'apparente similitude avec la composante principale de l'activité du mathématicien, celle de résolution des problèmes, la "démonstration " dans l'espace scolaire est fondée sur la formalisation - ou sur-sémiotisation pour suivre A. Mercier d'une mise en scène des savoirs mathématiques. Ainsi, la "démonstration " en tant

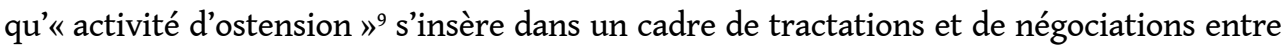
enseignants et élèves, dont la configuration est variable aussi bien selon ses effets sur la structuration institutionnelle des 88 savoirs que selon la composition sociale du public dans les groupes-classes.

Cette mise en scène de l'activité d'ostension mathématique a prioritairement pour objet de répondre aux «attentes » de l'enseignant. La notion d'« attente» (de l'enseignant), malgré son caractère le plus souvent peu spécifié, mérite à notre sens qu'on s'y arrête. Les «attentes» des enseignants, variables d'ailleurs selon les disciplines, se présentent en effet sous une forme complexe: variables selon la composition sociale du public ${ }^{10}$, elles condensent aussi bien les composantes du contrat didactique sous sa forme générique que les termes du contrat idiosyncrasique passé entre ceux-là et les élèves. Cela, dans l'hypothèse d'un contrat explicitement formulé en début d'année scolaire, ce qui, à notre connaissance, n'est pas toujours le cas. Il se peut en revanche que les règles d'interaction reposent sur des implicites, règles que les enseignants considèrent comme étant inscrites 
en amont dans les pratiques des élèves, ne nécessitant donc que de rectifications ou d'ajustements mineurs. C'est le cas de figure où les règles d'interaction sont considérées comme censées être acquises par les élèves, comme étant devenues une routine.

Routine des pratiques mettant en œuvre les règles d'interaction et négociations redéfinies en permanence s'opposent certes idéalement mais elles constituent, fort probablement à des degrés divers selon les moments de l'activité scolaire, les deux dimensions extrêmes qui, dans leur alternance, agencent et articulent les pratiques d'enseignement et les interprétations qui en sont faites aussi bien du point de vue de l'enseignant que du point de vue de l'élève ${ }^{11}$.

\section{Éducation scientifique et maîtrise linguistique}

11 Selon la séparation institutionnelle des disciplines scolaires, l'apprentissage de la langue - en l'occurrence le français - est limité à des heures précises, à des normes définies et à des corpus de connaissances particulières : langue et littérature.

Or, une maîtrise linguistique pluridimensionnelle est requise, nous semble-t-il, pour que les élèves puissent aborder l'acquisition-apprentissage des savoirs scientifiques. Elle ne se limite pas au seul « langage scientifique » ou au « langage mathématique » qui est prescrit par les programmes officiels, différemment d'ailleurs selon les disciplines. Cette maitrise repose sur un ensemble varié de compétences, présupposant la mobilisation simultanée de la part des élèves de plusieurs registres allant de la maîtrise de la langue comme moyen d'échange et de communication à la compréhension des éléments relevant du «langage scientifique».

13 Le décalage entre la transmission orale - le «cours » ou "l'explication » - et la forme écrite des «contrôles» et "examens» complique davantage les procédures de mobilisation de ces différents registres linguistiques et leur maitrise de la part des élèves. Certes, celle-ci fait partie - ou, du moins, on le souhaite -, de l'éducation scientifique. Mais elle est rarement explicitée dans sa globalité et les enseignants n'ont qu'une conscience approximative de la complexité de l'ensemble. Tout en mettant en œuvre une procédure de l'apprentissage de ce qu'ils considèrent comme « langage scientifique » ou « langage mathématique » proprement dit, par le biais souvent des listes des « conceptsnotions-mots " à faire apprendre, ils tendent à restreindre celui-là à ses dimensions étroitement formelles. Sont ainsi méconnus tous les autres registres linguistiques enchevêtrés dans la construction et reproduction (ou représentation) des savoirs scientifiques et techniques dans l'espace scolaire, tout autant que les spécificités dues au décalage entre présentations orales et présentations écrites. La compréhension des " énoncés » oraux ou écrits, la formulation des " problèmes ", des " démonstrations pour reprendre l'exemple des mathématiques, la «représentation» orale ou écrite des expériences en sciences physiques ou en technologie constituent autant de situations spécifiques présupposant la capacité des élèves à mobiliser plusieurs registres linguistiques en plus de la maîtrise du « langage scientifique » formellement défini. 


\section{NOTES}

1. Voir tout particulièrement l'ensemble de travaux effectués sous la direction de Jacqueline Eidelman, de l'équipe de sociologie de l'éducation, CNRS-Paris V.

2. C'est d'abord autour de la didactique des mathématiques que se sont formulées les premières théories de mise en perspective des savoirs scolaires du point de vue didactique (Guy Brousseau et Yves Chevallard), puis anthropologique (Yves Chevallard) et du point de vue psycho-génétique (Gérard Vergnaud).

3. Viviane Isambert-Jamati fut la première à analyser le passage du terme "connaissances » à celui de «savoirs" lors d'une conférence de séminaire de l'équipe de sociologie de l'éducation, CNRS-Paris V, en 1982.

4. L. Tanguy, F. Ropé, Savoirs et compétences. De l'usage social des notions à leur problématisation, Paris, L'Harmattan, 1994.

5. M.F.D. Young, «A Curriculum for the XXI ${ }^{\text {st }}$ Century? Towards a New Basis for Overcoming Academic/Vocational Divisions », British Journal of Educational Studies, August 1993.

6. Rappelons toutefois Gilbert Simondon, Du mode d'existence des objets techniques, Paris, Aubier, Éd. Montaigne, 1969 ; les travaux de Bertrand Gille et principalement l'Histoire des techniques, Paris, Gallimard, «Encyclopédie de la Pléiade», 1978; Jean-Claude Beaune, La Technologie introuvable. Recherche sur la définition et l'unité de la technologie à partir de quelques modèles du XVIII et XIX' siècles, Paris, Vrin, 1980 ou Gilbert Hottois, Le signe et la technique. La philosophie à l'épreuve de la technique, Paris, Aubier-Res, 1984.

7. Voir par exemple l'article de Philippe Nozières, professeur au Collège de France, dans le journal Le Monde, 11 octobre 1990, ou, plus récemment, Isabelle Stengers, La guerre des sciences, Paris, La Découverte, 1996.

8. Voir, par exemple, les travaux de B. Lahire sur «l'expression écrite » à l'école élémentaire : "L'inégalité devant la culture écrite scolaire : le cas de l'expression écrite à l'école primaire ", Sociétés Contemporaines, n¹1-12, 1992, nº́cial «Regards sur l'éducation " coordonné par L. Tanguy, mais aussi d'une façon plus large et plus problématisée les travaux de Françoise Ropé.

9. A. Mercier, «Le traitement public d'éléments privés du rapport des élèves aux objets de savoirs mathématiques ", in : Gilbert Arsac, Jean Gréa, Andrée Thiberghien, Denise Grenier (éds), Différents types de savoirs et leur articulation, Paris, La Pensée sauvage, 1995.

10. V. Isambert-Jamati, Culture technique et critique sociale à l'école élémentaire, Paris, PUF, 1984.

11. L'alternance dans le temps entre activités de routine et activités devenant objet de négociation entre catégories d'acteurs n'est pas spécifique aux pratiques d'enseignement. Cette articulation peut être repérée dans l'exercice de toutes les activités professionnelles.

\section{RÉSUMÉS}

Le découpage institutionnel qui trouve sa traduction dans l'opposition entre sciences et technologie est aussi présent dans l'apprentissage du langage. Pour transmettre des résultats 
scientifiques, les élèves font appel à plusieurs registres de langue ; seul le langage spécialisé fait l'objet, dans le cadre de l'éducation scientifique, d'un apprentissage se limitant souvent à l'acquisition de vocabulaire, les autres registres étant ignorés dans ce cadre.

INDEX

Index géographique : France

Mots-clés : éducation scientifique, études scientifiques, langue de spécialité, langue scientifique

\section{AUTEUR}

POLYMNIA ZAGEFKA

Maître de conférences, Centre de recherches sociologiques et historiques sur l'éducation, École normale supérieure de Fontenay-Saint-Cloud, France. 